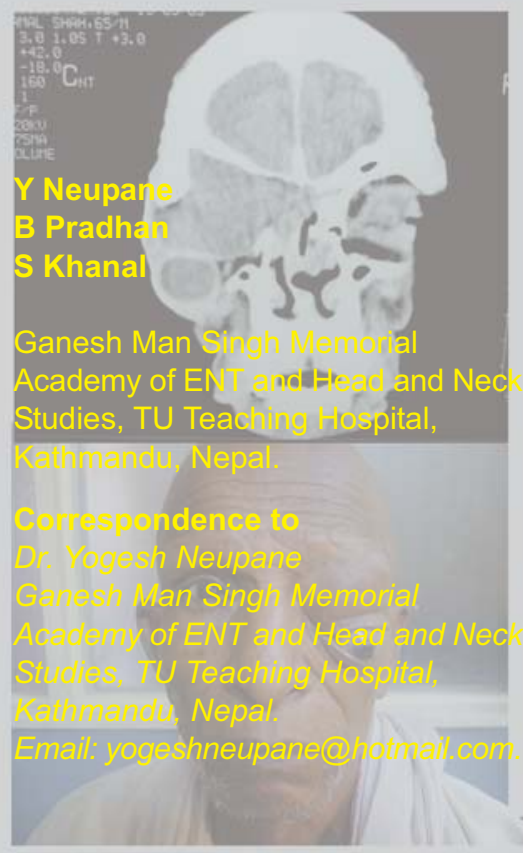

\title{
An unusual Presentation of Fronto-Ethmoidal Mucocele
}

An unusual presentation of fronto-ethmoidal mucocele is reported. A 65 years old male presented with complains of painless swelling over left supraorbital region, protrusion of left eye ball for 6 months and loss of vision left eye for 4 months. On examination there was swelling over left supraorbital region with nonaxial proptosis. CT scan showed well defined homogenous soft tissue density in left orbit, frontal and ethmoid sinuses displacing eyeball forward, downward and laterally. Endoscopic marsupialization of mucocele with fronto-ethmiodectomy was performed.

Key words: Fronto-ethmoidal mucocele, Endoscopic, Marsupialization

INTRODUCTION:

A mucocele is an epithelial lined, mucous containing sac completely filling the sinus and capable of expansion. ${ }^{1}$ This is in contradistinction to a blocked sinus cavity which simply contains mucus. ${ }^{2}$ Fronto-ethmoidal mucocele is by far the most common, maxillary being the least common type. Frontoethmoidal area is more susceptible to mucocele formation due to complexity of its drainage as compared to other sinus. ${ }^{1}$ Frontoethmoidal mucocele usually present with outward and downward displacement of orbital globe and are often associated with palpable mass in supero-nasal and medial canthal region. The main objective of this case report is to report the rare presentation of fronto-ethmoidal mucocele.

\section{CASE REPORT:}

A 65 years old male presented with complains of swelling over left supraorbital region, forward protrusion of left eye ball for 6 months and loss of vision left eye for 4 months. He gave history of trauma over left supraorbital region 2 years back. Since 6 months he noticed swelling over medial aspect of left supraorbital region which was insidious, gradually progressive and painless. He gave history of decreased vision in left eye since 2 years which was gradual and progressive and complete loss of vision since 4 months. On examination there was $6 \times 5 \mathrm{~cm}$ single, smooth, globular swelling extending superiorly $1 \mathrm{~cm}$ above supraorbital rim, inferiorly $2 \mathrm{~cm}$ below infraorbital rim, medially $0.5 \mathrm{~cm}$ lateral to medial canthus (Fig.1). Swelling was nontender, firm to hard, nonpulsatile, fixed to underlying structure but overlying skin was free. Swelling was pushing the eyeball downward, forward and outward. On ophthalmic examination there was non axial proptosis, protruding $2 \mathrm{~mm}$ anteriorly with $2 \mathrm{~cm}$ downward and $2 \mathrm{~cm}$ outward displacement. Movements of extraocular muscles were restricted in all gaze, conjunctiva was

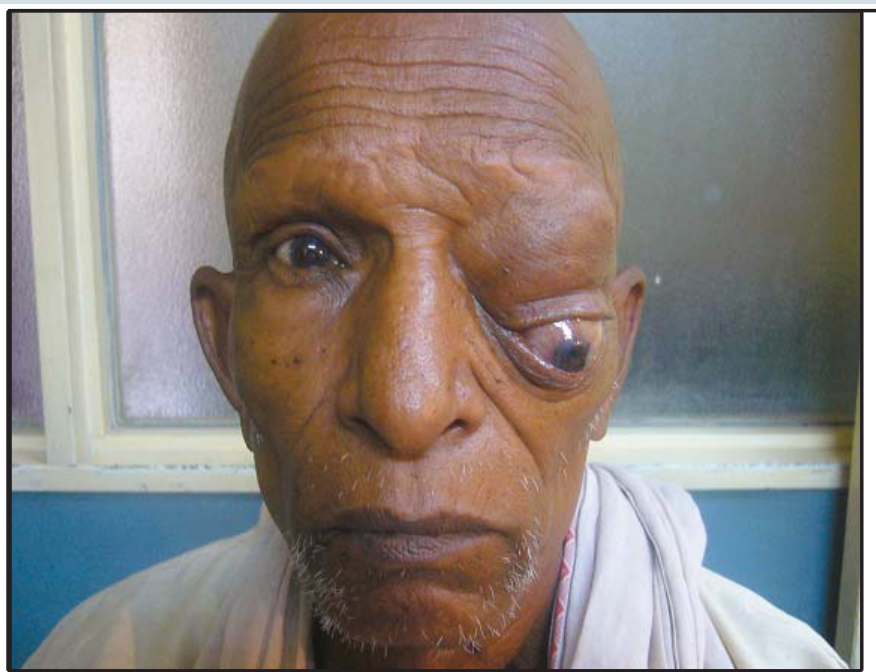

Fig.1: Showing swelling \& proptosis

congested and corneal examination showed feature of exposure keratisis. Anterior chamber was quiet, pupil was sluggishly reacting, lens were cataractous (Left $>$ Right). However, fundus couldn't be visualized. Sensation over cheek was intact but infraorbital rim was blunted. Anterior rhinoscopy was normal. Left nasal endoscopy showed $0.5 \times 0.5 \mathrm{~cm}$ small round mass lateral to medial turbinate and accessory maxillary ostium was present. Fine needle aspiration cytology from swelling showed few scattered foamy macrophages and few inflammatory cells with thick mucinous material in background compatible with mucocele. Computed tomography scan showed well defined homogenous soft tissue density in left orbit (Fig. 2). Superiorly the frontal sinus was not visualized and superior wall of sinus lifted up and thinned out on its medial aspect. Laterally, lateral wall of ethmoid was not eroded but the mass extended into 


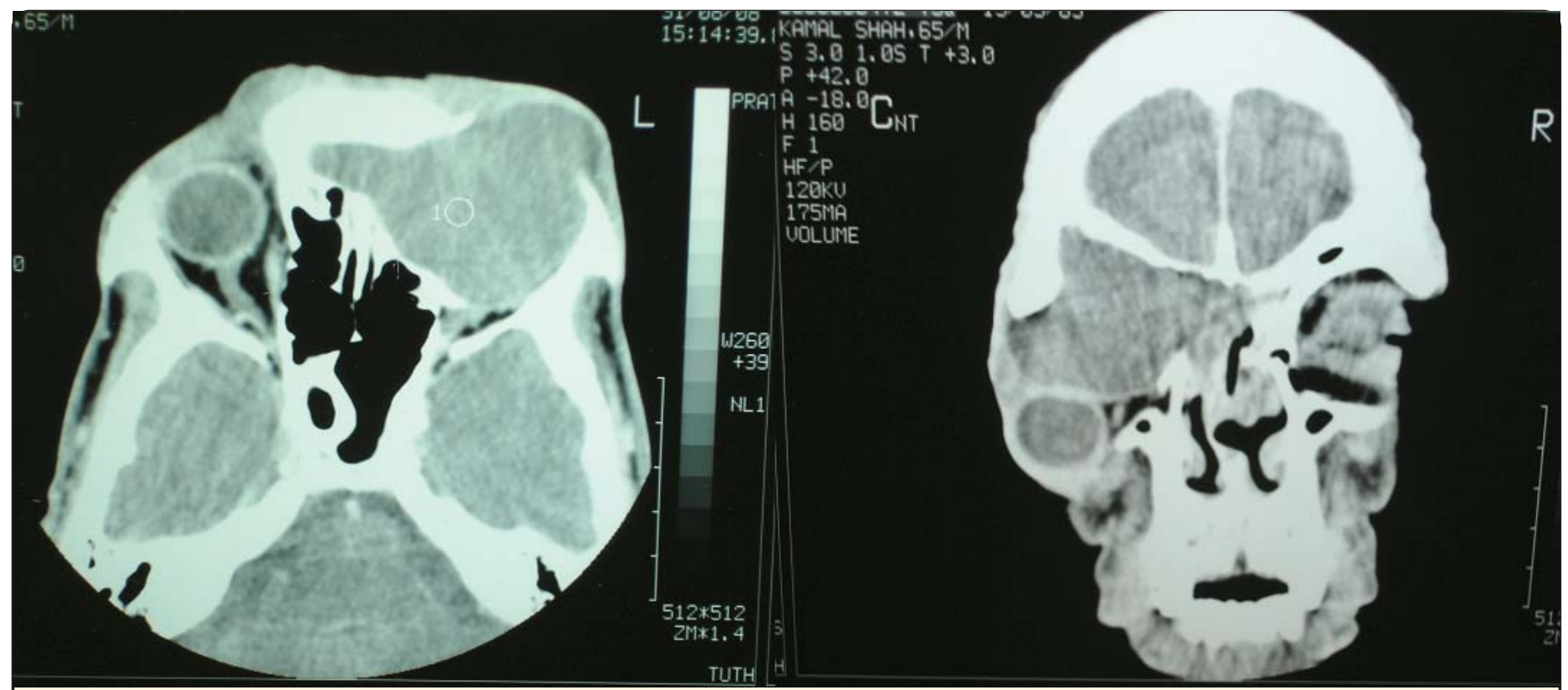

Fig.2: Axial and coronal CT scan of nose and PNS showing soft tissue mass noted in left orbit pushing orbital globe infero-laterally

nasal cavity. Inferiorly floor was pushed downward and was thinned out. The globe was pushed inferiorly and outward along extra ocular muscles. The optic nerve sheath was displaced inferiorly by the mass. After Intravenous contrast there was peripheral (wall) enhancement. Orbital apex and optic foramen were normal.Based on clinical findings and investigation, the most likely diagnosis was frontal mucocele with left cataract. The diagnosis of frontal mucocele was confirmed at surgery. Endoscopic wide marsupialization of mucocele with left frontoethmoidectomy was performed under general anaesthesia. Peroperatively about $100 \mathrm{ml}$ of thick tenacious green-yellowish fluid was obtained after puncturing the bulla. Anterior table and part of posterior table of left frontal sinus was eroded. Dura could be seen through defect in posterior wall of frontal sinus but was found to be intact. Post operatively there was rapid resolution of swelling with improvement of proptosis and eye movement (Fig.3). Culture of aspirated fluid revealed no growth

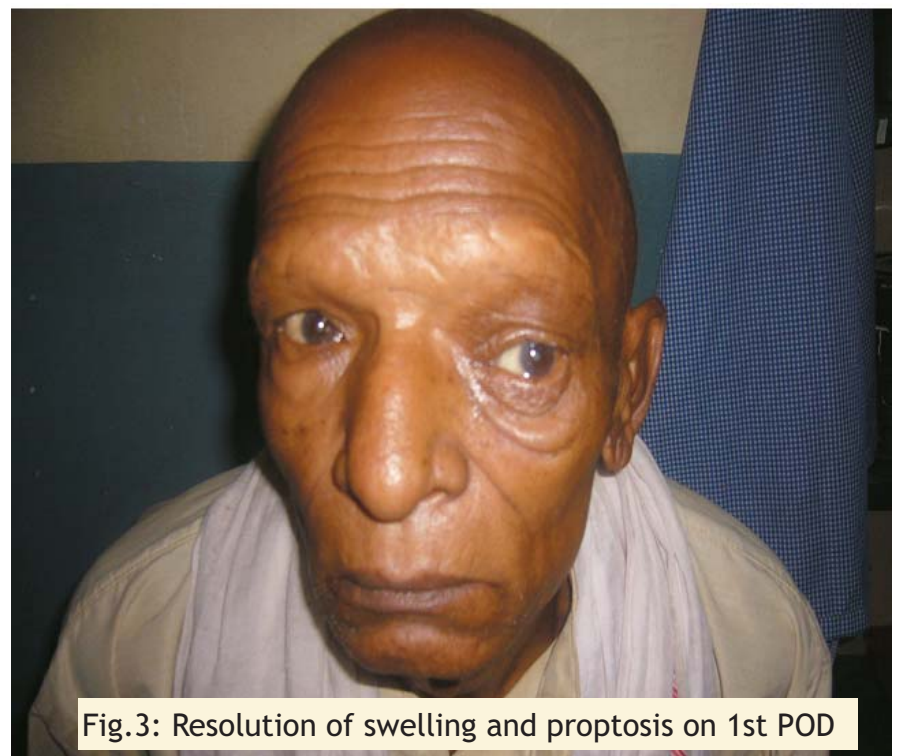

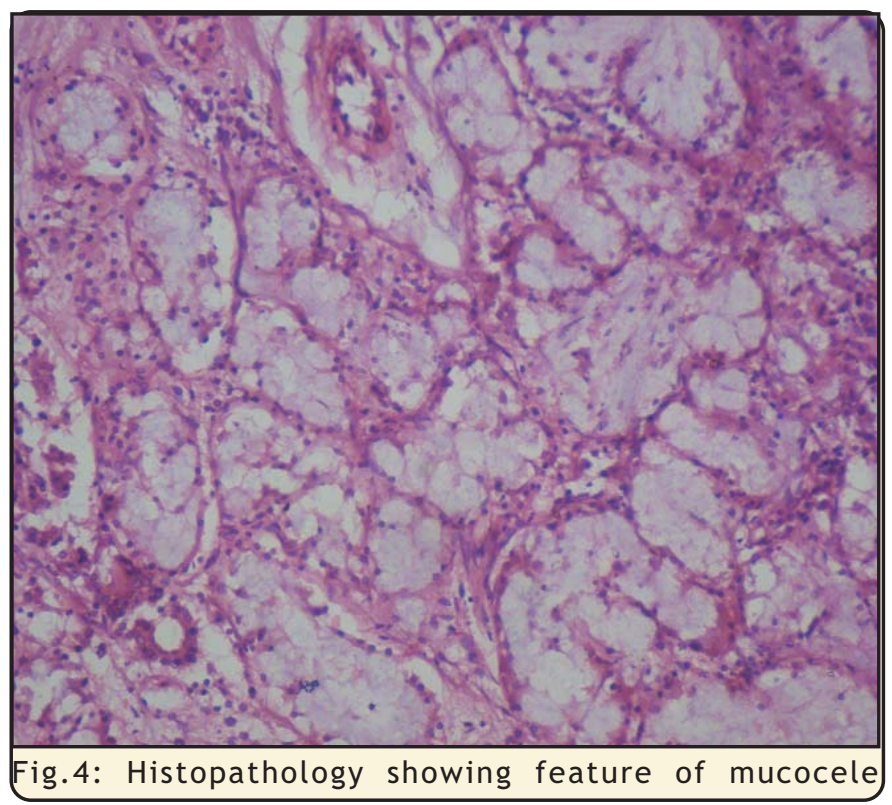

after 72 hours of incubation. Mucosa was studied histologically, the finding of which were consistent with the diagnosis of mucocele (Fig. 4). The patient made an uneventful post operative recovery. Nasal endoscopy four months after surgery showed widely open sinus cavity with healthy epithelization with no sign of recurrence. He was planned for cataract surgery on later date.

DISCUSSION:

The common presenting feature of fronto-ethmoidal mucocele are orbital displacement, frontoethmoidal swelling, visual impairment, headache, fistula in same region if spontaneous bursting spontaneously or any attempts is made to drain it. In a case series of 120 patients with fronto-ethmoidal mucocele $95 \%$ had some degree of proptosis, $55 \%$ had lateral displacement, and $59 \%$ had inferior displacement. Ocular mobility was limited 
in $55 \%$ in upgaze by mass which was often palpable in upper medial quadrant of orbit. ${ }^{3}$ Similarly Tseng et al. found proptosis as most common presentation in patient with fronto-ethmoidal mucocele. ${ }^{4}$ Nasal endoscopy may reveal the expanded mass presenting in ethmoidal region. However our patient didn't give the history of headache or nasal obstruction. He gave history of swelling over supraorbital region instead over frontal or orbital region which is rare in itself as presenting feature of frontoethmoidal mucocele. Two third of mucocele are secondary to obvious predisposing factors and one third are idiopathic. Mucoceles are unilateral in $95 \%$ and bilateral in $5 \%$ of cases. ${ }^{5}$ Time lag between clinical presentations and initiating factor of mucocele varies. In case of surgery or trauma this is in an average of 23 years, whereas following an acute infective episode the mean time for presentation is 22 months. Five our patient had history of trauma two years back. There are various theories in pathogenesis of mucocele like pressure erosion, cystic degeneration of glandular tissue and active bone resorption and regeneration. Active bone resorption and regeneration theories are supported by greater number of evidence. A number of boneresorbing factors have been found in mucocele mucosa like PGE2, leukotrienes, HETES and a range of cytokines. ${ }^{6}$ Also increased level of IL-a, IL1B and TNF-a, vascular adhesion molecules, e-selectin and I-CAM are found in mucocele lining in comparison with normal controls. ${ }^{7}$ In normal situation, new bone formation is balanced by osteolysis. In mucocele, the balanced is just tipped in favor of osteolysis facilitating expansion of lesion .Computed tomography scan is optimum method of demonstrating a mucocele. ${ }^{8}$ Several treatment options are available and choice depends on the degree of extension ${ }^{9}$ and may range from functional endoscopic sinus surgery to external approach, craniotomy and craniofacial exposure with or without obliteration of the sinus. ${ }^{10}$ The current tendency is to conduct functional, minimal invasive and low morbidity procedure with nasosinusal endoscopic surgery with marsupialization and abundant drainage of the lesion, preserving the epithelium. ${ }^{11}$ Frontoethmoidal mucocele are commonest among the mucocele occurring in various paranasal sinuses and may present in various way. Though rare but supraorbital subcutaneous soft tissue mass may be presenting complaint. So careful examination and investigation may suggest the diagnosis of sinus related disease.

\section{REFERENCES:}

1. Natvig K, Larsen TE. Mucocele of paranasal sinuses. Journal of Laryngology and otology.1978; 92: 1075-082.

2. Schenck NL, Rauchbach E, Ogura JH. Fronto sinus disease,II. Development of the frontal

sinus model: Occlusion of Nasofrontal duct. Laryngoscope 1974; 84: 1233-47.

3. Valerie J Lund. Mucoceles (119). Scott-Brown's Otorhinolaryngology, Head and neck surgery, 7th edn. London: Hodder Arnold, 2008 : 1531-38

4. Chih-Chen Tseng, Ching-yin Ho, Shu-Ching Kao.ophthalmic manifestation of paranasal sinus mucoceles.journal of Chinese medical association .2005:68(6); 260-264

5. Lund VJ. Anatomical considerations in the aetiology of fronto-ethmoidal mucoceles. Rhinology 1987; 25: 83-88.

6. Lund VJ, Harvey W, Meghji S, Harris M. Prostaglandin synthesis in the pathogenesis of fronto ethmoidal mucoceles. Acta Otolaryngol. 1988; 106: 145-51.

7. Lund VJ, Henderson B, Jong Y. Involvement of cytokines and vascular adhesion receptors in the pathology of frontoethmoidal mucocele. Acta Otollaryngol. 1998; 113; 540-5

8. Kennedy DW, Josephson JS, Zinreich SJ, Matrox DE, Goldsmith MM. Endoscopic sinus surgery for mucoceles. Laryngoscope .1989; 99: 885-95

9. Koike Y, Takara K. Chiha Y, Suzuki SI, Mlirai M, Ita H. Intracranial extension of paranasal sinus mucocele: two case reports. Surg neurol J.1996; 45: 44-8.

10. Weitzel EK, Haller LH, Calzada G, Manalids S. Single stage management of complex fronto-orbital mucocele. J craniofac Surg. 2002; 13: 739-45

11. Bussaba NY, Salman SB, Maxillary sinus mucoceles: clinical presentation and long term results of endoscopic surgical treatment. The laryngoscocpe.1999: 1446-9 